\title{
Control on-off de temperatura y potencia para el mejoramiento de las condiciones de procesos asistidos con microondas
}

\section{On-off temperature and power controller for improvement of the processes conditions assisted with microwaves}

\author{
DOI: http://doi.org/10.17981/ingecuc.13.2.2017.06
}

Artículo de investigación científica. Fecha de recepción: 26 de septiembre de 2016 - Fecha de aceptación: 25 de junio de 2017.

\section{Viviana Marcela Hernández Velásquez}

Servicio Nacional de Aprendizaje SENA. Medellín (Colombia). vivimhernandez@gmail.com

\section{María Bernarda Alvarado Bawab}

Institución Universitaria ITSA. Soledad (Colombia). maryalvaradoiqad@gmail.com

Para citar este artículo:

V. Hernández Velásquez y M. Alvarado Bawab. "Control on-off de temperatura y potencia para el mejoramiento de las condiciones de procesos asistido con microondas" INGE CUC, vol. 13, no. 2, pp. 53-59, 2017 DOI: http://doi.org/10.17981/ingecuc.13.2.2017.06

\section{Resumen}

Introducción- El uso de microondas en el proceso de deshidratación de frutas $\mathrm{y}$ verduras se presenta como un proceso alternativo a los convencionales debido a que ofrece beneficios en cuanto a la reducción de costos de transporte, menor tiempo de procesamiento y volumen del producto final y mayor tiempo de conservación y almacenamiento.

Objetivo- Este estudio busca modificar la forma de suministro de la radiación de microondas, implementando un control on-off de potencia y temperatura a fin de evaluar estos parámetros tanto en el rendimiento del proceso de la deshidratación de la papaya como en su rendimiento energético.

Metodología- Para el desarrollo del proyecto se realizó un diseño factorial de experimentos teniendo en cuenta el tiempo de encendido y apagado de la radiación y la geometría de la muestra (torreja y cubo). Se realizaron las corridas por duplicado y de forma aleatorizada en el horno microondas de $2,45 \mathrm{GHz}$ y $1 \mathrm{~kW}$ de potencia modificado.

Resultados-Se analizó la cantidad de humedad removida, el rendimiento energético del proceso y las propiedades organolépticas iniciales. En las pruebas realizadas se logró un máximo rendimiento que se puso en evidencia en el proceso de secado de papaya, el cual fue de $0,014 \mathrm{~kg} / \mathrm{kJ}$ con una reducción del $86 \%$ de peso de la muestra procesada en la razón de radiación de 6 x 12 para una rodaja de $0,01 \mathrm{~m}$ de espesor.

Conclusiones-Se logró la modificación del control en el horno microondas y de las corridas realizadas y se pudo concluir que los parámetros evaluados si son influyentes en el proceso y se pueden alcanzar remociones de humedad hasta del $86 \%$ sin deteriorar la papaya cuando se aplica la razón de radiación adecuada.

Palabras clave- Controlador, microondas, potencia, temporizado, temperatura, horno, rendimiento, proceso.

\section{Abstract}

Introduction- The use of microwaves in the process of fruits and vegetables dehydration is presented as an alternative process to the conventional ones because the benefits in a reduction of transport costs, less processing time and final product volume, as well as greater time of conservation and storage.

Objective- Therefore, the aim of this study is to modify the microwave radiation supply, implementing an ON-OFF control of power and temperature in order to evaluate these parameters in the process and energy yield in the papaya.

Methodology- For the development of the project, a factorial design of experiments was done taking into account the time on and off of the radiation and the sample geometry (slice and cube); runs were performed in duplicate and randomly in the modified microwave oven of $2.45 \mathrm{GHz}$ and $1 \mathrm{~kW}$ of power.

Results- The amount of moisture removed, the energy yield of the process and the initial organoleptic properties were analyzed. In the tests carried out, a maximum energy yield was $0.014 \mathrm{~kg} / \mathrm{kJ}$ with a reduction of $86 \%$ of the papaya sample weight processed in the radiation rate of $6 \times 12$ for a slice of $0,01 \mathrm{~m}$ of thickness.

Conclusions- It was achieved modification of the control in the microwave oven and the runs were carried out concluding that the parameters evaluated and are influential in the process and can be achieved moisture removal of $86 \%$.

Keywords- Controller, Microwave, Power, Timing, Temperature. 


\section{INTRODUCTION}

Las aplicaciones de la energía de microondas han sido ampliamente estudiadas en las últimas décadas en un amplio espectro, reportando como resultados una exitosa mejora en los experimentos con respecto a los métodos convencionales. La mayoría de los estudios llevados a cabo con energía de microondas se han centrado principalmente en su capacidad de calentamiento debido a la transferencia de energía de forma directa hacia el material, permitiendo un calentamiento rápido y volumétrico de la muestra, mientras evita complicaciones como los amplios gradientes de temperatura entre la superficie y el interior, tal como se presenta en los métodos convencionales [1].

El calentamiento volumétrico de las muestras va acompañado por la capacidad de la energía de microondas para penetrar el interior de la muestra e inducir un calentamiento más profundo en la muestra. Lo anterior se debe a que la energía de microondas a través de los campos eléctricos y magnéticos influye en las muestras; las moléculas polares e iónicas presentes tratan de alinearse con el campo eléctrico oscilante, generando calor por fricción debido a que los gradientes iónicos cambian constantemente y las partículas colisionan.

La naturaleza de la energía de microondas para calentar selectivamente materiales dieléctricos hace evidente la ventaja de su uso en el secado de materiales. La mayoría de las investigaciones en esta aplicación se centran en la preservación de alimentos como pastas [2], frutas [3], verduras y aperitivos salados [4] a través de la deshidratación.

Otros procesos asistidos por microondas empleados a escala, tanto de laboratorio como industrial son: el proceso de pirólisis [5], [6], la pasteurización [7] y la cocción de alimentos [8], en los cuales se han evaluado principalmente las variables de operación y propiedades de los materiales sometidos a los procesos. Por tal razón, el proyecto presentado en este artículo busca desarrollar un control on-off en el que se considere la intermitencia de aplicación de la radiación microondas y garantice el uso eficiente de la energía sin afectar las propiedades físico-químicas del material procesado.

El secado asistido por microondas ofrece ventajas sobre los secadores convencionales de aire caliente, tales como: tiempos de secado más cortos, aumento de la eficiencia energética y potencial de la reducción del tamaño de los equipos de secado requeridos [9]. De igual forma, se hace interesante, y aún más eficaz, la combinación con procesos convencionales como el secado con aire caliente [9]. La deshidratación de frutas y verduras es un proceso muy conveniente para optimizar su vida útil, manteniendo el valor nutritivo y la calidad. Las características comúnmente elegidas para repro- char el secado asistido por microondas de frutas y verduras son generalmente el tiempo de secado y la calidad del producto seco (textura, dureza, color de la superficie, la concentración de vitaminas, la capacidad de rehidratación, y lo más importante, el sabor) [10]. Sin embargo, es necesario realizar pruebas experimentales para definir los rangos de operación y el suministro adecuado de la radiación microondas.

Con el pasar del tiempo se observa un aumento en el uso de las tecnologías de microondas para procesos de secado con diferentes productos industriales y alimenticios, donde se ha encontrado una importante disminución no solo en el uso de la energía sino en el tiempo de secado del producto seleccionadoevidenciancia del manual.su funcionamiento desde la lcomponentes y l.acionalesel proyecto. [2]. Sin embargo, este es un avance que en Colombia aún está en una fase de estudio, por tal motivo, y observando que en el momento los hornos microondas convencionales tienen definidos los ciclos de funcionamiento o de potencia (lo cual no garantiza un secado controlado y, por ende, no es posible controlar o mantener las condiciones del producto a secar), este trabajo diseña e implementa, en un horno microondas de $1 \mathrm{~kW}$ de potencia a la frecuencia de $2,45 \mathrm{GHz}$, un programa que permite manipular la exposición del proceso a una determinada temperatura mediante la acción de un control on-off.

\section{Revisión Literaria}

Un sistema de control consiste en captar información del ambiente a controlar a través de componentes físicos que miden distintas variables, y cuya información obtenida es transmitida hacia el controlador programable para la toma de decisiones. Los sensores pueden ser de tipo análogo o digital y se conectan a las entradas de los PLC [11]. Los sistemas de tratamiento térmico de materiales utilizando energía de microondas vienen utilizándose durante más de 60 años. La técnica en el diseño e implementación de este tipo de sistemas ha avanzado enormemente durante este tiempo, pero el control preciso de la temperatura de los materiales sigue presentando muchas dificultades teóricas y prácticas. En el proceso estudiado sobre el secado de carbón asistido con microondas, se logró establecer a través de simulaciones y pruebas experimentales que la relación masa-potencia, la altura del lecho, la humedad, la composición y el tiempo de tratamiento son variables a controlar para obtener los resultados esperados. La razón de radiación, entendida como un control on/off que domine la intermitencia en que se suministra la onda al horno microondas es el parámetro más importante para mejorar tanto los rendimientos energéticos como los del proceso [12]. 
Otra de las formas de mejorar el sistema de control en los equipos de microondas consiste en modificar los tipos de aplicadores de microondas que se pueden utilizar (unimodal, multimodal), así como las técnicas de optimización de la distribución de temperatura aplicables a cada tipo de aplicador en función del campo eléctrico que producen los agitadores de modos. Otro aspecto importante a estudiar para el sistema de control es el tipo de sensor de temperatura y su aplicabilidad a los sistemas de microondas, ya que el incremento de la temperatura es determinado por la potencia absorbida [13].

En el estudio estadístico del proceso de secado de carbón se encontró que la temperatura no debe superar los $90^{\circ} \mathrm{C}$ y el nivel 5 es el máximo nivel de potencia a emplear en un horno convencional microondas de $1 \mathrm{~kW}$ [14]. Lo anterior corresponde a una razón de radiación de 12 segundos de encendido por 10 de apagado, en un tiempo total de ciclo de 22 segundos que está predeterminado en los hornos microondas comerciales. Con el objeto de estudiar diferentes razones de radiación, manipularlas manualmente y conocer en tiempo real la temperatura alcanzada a fin de prevenir efectos no deseados sobre la muestra, se propone el equipo que se presenta en este artículo.

En todos los procesos es indispensable controlar la forma de suministro de energía a fin de evaluar las condiciones y forma cómo se transporta el calor y la materia; no conocer en tiempo real las variables claves como temperatura ni poder controlarla durante un calentamiento que conlleva al secado de un material poroso hace que no sea posible estandarizar el proceso ni garantizar el producto final ya que puede ser deteriorado por fracturas, craqueo, extracción de volátiles, quemado, etc., situaciones muy comunes en los procesos de calentamiento por microondas al ser un proceso dinámico que evoluciona según la muestra cambie de temperatura y sus propiedades en función de ésta.

\section{III.METODOLOGÍA}

\section{A. Horno microondas LG MS 0746T}

El equipo empleado como base para la modificación de adecuación del control diseñado es un horno microondas marca LG modelo MS 0746T. Inicialmente, se estudió su funcionamiento desde la lógica eléctrica en cuanto a las especificaciones para el manejo de la potencia del equipo; se estudió el diagrama eléctrico, el cual se presenta en la fig. 1, obtenido del manual de servicio del equipo [15] con el fin de hacer una adecuada distribución de las salidas de la tarjeta electrónica diseñada.

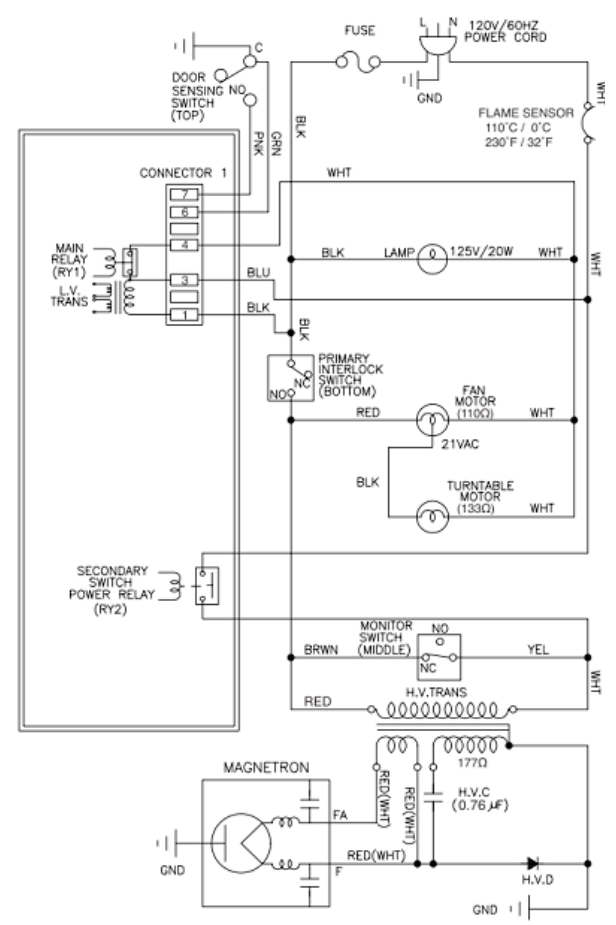

Fig. 1. Diagrama de conexiones eléctricas microondas MS-0746T Fuente: [15].

Este diagrama muestra las conexiones que tiene el equipo y, principalmente, las borneras donde está conectado el transformador y, por ende, el magnetrón. Lo anterior es necesario para tomarse como base y proponer el control on-off y la medición de la temperatura a fin de optimizar el proceso; para realizar esto, se aprovechó la instalación del equipo y solo se modificó la tarjeta electrónica con el control diseñado propuesto.

Una vez obtenido el prototipo del horno microondas con la capacidad de seleccionar las variables propuestas en este artículo, se realizaron las corridas experimentales a muestras de 50 gr de papaya, controlando la geometría de las muestras en cubos de $0.01 \mathrm{~m}$ y torrejas del mismo alto y utilizando bandejas para introducirlas en el horno microondas. Se realizaron las pruebas a razón de radiación de 3x3, 3x6, 3x9, 6x6 y $6 \times 12$; en las cuales se puso en evidencia la razón de radiación adecuada para el desarrollo de las actividades de deshidratación de la papaya.

Para garantizar la igualdad entre los procesos, se tuvo en cuenta que el tiempo total de radiación de microondas suministrada a la muestra fuera de $600 \mathrm{~s}$, se registró la temperatura cada minuto durante todo el tiempo de operación y los cambios físicos observados en las muestras.

Con los datos obtenidos se realizaron y analizaron las curvas de secado, se identificaron límites de operación para cada condición estipulada y se evaluó el rendimiento energético de cada condición de proceso. 


\section{Resultados}

\section{A. Diseño de modo de funcionamiento}

Para el diseño del control del horno microondas, se especificó la necesidad de diferenciar dos modos de operación. El primer modo (modo manual) tiene en cuenta los tiempos de encendido y apagado del magnetrón en el horno microondas, con lo que se puede elegir qué totalidad de tiempo se requiere para el proceso y el ciclo útil del equipo; y el segundo modo (modo automático) condiciona el proceso operando bajo un rango de temperaturas que determina la decisión de mantener apagado o encendido el suministro de radiación de microondas a la cámara de procesamiento donde se encuentra el material a tratar.

\section{1) Modo manual}

Este primer modo de funcionamiento manipula el tiempo del ciclo útil del magnetrón, es decir, el usuario puede seleccionar el tiempo que desea que el producto esté expuesto a la radiación de microondas, por tanto, podrá seleccionar los requerimientos que el programa pedirá al usuario para poder iniciar la asignación de las siguientes condiciones:

- Tiempo de ciclo alto

- Tiempo de ciclo bajo

- Tiempo total

La temperatura se registra en todo momento, pero el equipo no tiene en cuenta esta variable para detener el proceso, solo se detendrá el proceso cuando haya pasado el tiempo total elegido o se pulse el botón de stop, el cual es un botón de emergencia para detener el proceso.

\section{2) Modo automático}

El modo de funcionamiento automático requiere que se tenga un control de temperatura, es decir, el usuario selecciona cuál es el rango de temperatura a la que el equipo debe llegar. Debido a esa condición, el equipo mantendrá dicho rango de temperatura para que el proceso se lleve a cabo bajo las condiciones deseadas. Los requerimientos para este modo de funcionamiento son:

- Rango de temperatura máxima

- Rango de temperatura mínima

- Tiempo total

El control elegido para esta primera etapa es un control on-off, ya que el proceso de temperatura se hace un poco lento y es más eficiente mantener esta variable en una condición específica con las fluctuaciones de las microondas dentro de la cavidad sin afectar las condiciones del producto procesado. El esquema de control diseñado se presenta en la fig. 2.

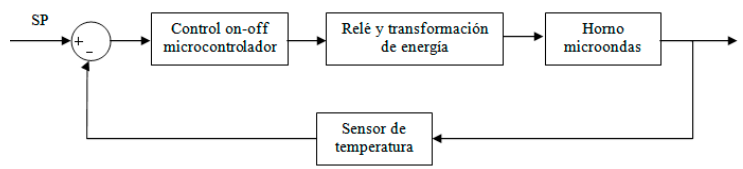

Fig. 2. Diagrama de bloques del proceso. Fuente: Elaboración propia.

El diagrama de bloques describe los componentes utilizados y la utilidad dentro del proceso, por tanto, los instrumentos usados son un sensor de temperatura que cubre un rango de entre 0 y $350^{\circ} \mathrm{C}$; el controlador, un microprocesador PIC18F4550; y el actuador, un relé que permite habilitar la señal hacia el magnetrón desde el controlador.

\section{Diseño de la tarjeta electrónica}

Teniendo en cuenta los dos modos de funcionamiento y el diseño de la tarjeta de fábrica del equipo $(179,18$ $\mathrm{mm} \times 80,00 \mathrm{~mm}$ ), se diseñó una tarjeta que cumple con las especificaciones del equipo, tanto en control como de las conversiones necesarias a los voltajes desde AC a DC y DC a AC, con los cuales se garantiza un adecuado funcionamiento de los instrumentos usados para generar las microondas. Para el diseño de la tarjeta se utilizó un software llamado Altium designer, que permite hacer el diseño profesional de tarjetas electrónicas con la posibilidad de visualizar diferentes perspectivas del diseño, así como se puede observar en la fig. 3 .
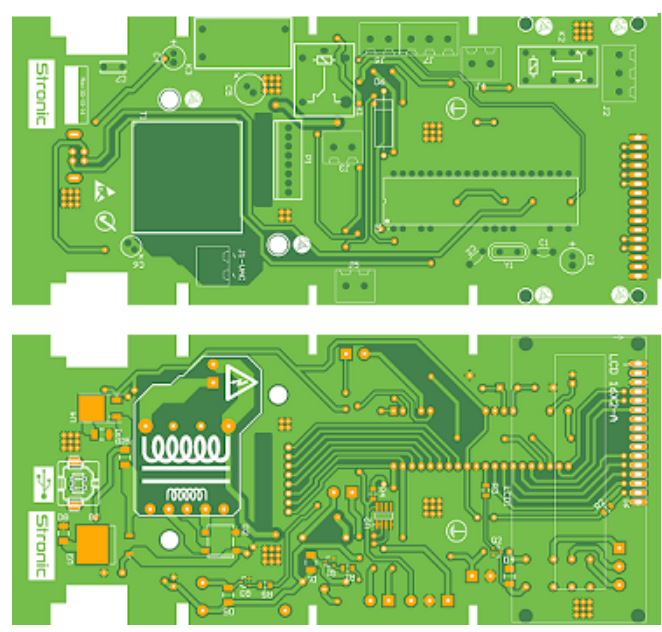

Fig. 3. Bosquejo de tarjeta electrónica de microondas. Fuente: Elaboración propia.

Esta tarjeta contiene los elementos necesarios para acondicionar el voltaje, leer el sensor de temperatura, el teclado y, por ende, mostrar en el display cada modo de funcionamiento, el cual llevará al usuario a elegir las condiciones de operación de la opción deseada. 
Las microondas fueron controladas para sistemas de radar en la Segunda Guerra Mundial en el año 1946, y unos años más tarde apareció el horno microondas en el mercado. Los procesos asistidos con microondas poseen características diferentes a las que experimentan los materiales en los procesos por métodos convencionales, entre ellos: la capacidad de penetración de la radiación, lo que permite un calentamiento rápido y volumétrico; evitar el calentamiento de un recipiente o medio que transporte la energía; la selectividad de los materiales a calentar; la capacidad de controlar la distribución del calor en determinadas zonas, entre otras. Por tanto, uno de los procesos industriales atractivos para emplear esta tecnología es el secado, el cual es empleado en el procesamiento de arcillas, alimentos, sustancias químicas, polímeros y algunos materiales compuestos.

Las ventajas que se estudian con el uso de los procesos asistidos por microondas se basan principalmente en el ahorro del tiempo de procesamiento de la materia, el aumento de la productividad y la reducción de los niveles de contaminación al ambiente, buscando así ser un complemento que permita obtener resultados óptimos sin afectar la composición y propiedades del material a procesar.

\section{Corridas experimentales}

De acuerdo con las variables objeto de estudio en este trabajo, se identificó la geometría de la muestra y la razón de radiación. Se analizó la cantidad de humedad removida, el rendimiento energético del proceso y las propiedades organolépticas iniciales. En la Tabla 1 se presentan los resultados obtenidos en cada uno de los procesos realizados para las muestras de $50 \mathrm{~g}$ en cubos de $0.01 \mathrm{~m}$ y rodajas de $0.01 \mathrm{~m}$ de alto.

En esta tabla se presentan los tiempos límites máximos de operación para cada una de las condiciones sin presentar deterioro en las muestras, de allí la variación en los tiempos de tratamiento y energía total suministrada.

De las razones de radiación seleccionadas objeto de estudio se pone en evidencia que el tiempo de encendido de 6 segundos presenta mejores rendimientos energéticos que los de 3 segundos. Los tiempos de 6 segundos de encendido además presentaron pérdidas de peso de la muestra semejantes a algunos procesos llevados a cabo con 3 segundos de encendido en la razón de radiación, pero en alrededor de la mitad del tiempo de proceso.

Por otra parte, tiempos de reposo mayores presentan mejores rendimientos, tanto del secado como energético, aunque existe un límite. Lo anterior se explica porque en el tiempo donde no es irradiada la muestra con microondas se permite la conducción del calor favoreciendo una distribución uniforme de la energía en el interior de la muestra, previniendo puntos calientes y la difusión uniforme de la humedad hacia el ambiente. Se puede inferir que la temperatura ideal de procesamiento se encuentra entre los 50 y $70^{\circ} \mathrm{C}$.

TABla 1. Resultados DEL SECAdo DE PAPAYA ASISTIDO POR RAZONES DE RADIACIÓN DE MICROONDAS DE $2,45 \mathrm{GHz}$ Y $1 \mathrm{~K}$ W DE POTENCIA.

\begin{tabular}{|c|c|c|c|c|c|c|}
\hline $\begin{array}{c}\text { Razón de } \\
\text { radiación }\end{array}$ & Muestra & $\begin{array}{c}\text { Tiempo tratamiento } \\
(\mathrm{min})\end{array}$ & $\begin{array}{c}\text { \% Perdida de } \\
\text { peso total }\end{array}$ & $\begin{array}{c}\text { Energía } \\
\text { suministrada (kJ) }\end{array}$ & $\begin{array}{c}\text { Temperatura } \\
\text { promedio (K) }\end{array}$ & $\begin{array}{c}\text { Rendimiento } \\
(\mathrm{kg} / \mathrm{kJ})\end{array}$ \\
\hline $6 \times 12$ & Cubo & 15 & 65,42 & 300 & 335 & 0,01090 \\
\hline & Torreja & & 86,24 & & 343 & 0,01437 \\
\hline $6 \times 6$ & Cubo & 12 & 63,43 & 360 & 338 & 0,00881 \\
\hline & Torreja & & 66,38 & & 337 & 0,00922 \\
\hline $3 \times 9$ & Cubo & 32 & 54,59 & 480 & 326 & 0,00569 \\
\hline $3 \times 6$ & Torreja & & 82,13 & & 337 & 0,00855 \\
\hline & Cubo & 30 & 63,93 & 600 & 331 & 0,00533 \\
\hline $3 \times 12$ & Cubo & 50 & 76,77 & & 335 & 0,00640 \\
\hline & Torreja & & 51,14 & 600 & 323 & 0,00426 \\
\hline $3 \times 3$ & Cubo & 12 & 59,54 & & 331 & 0,00496 \\
\hline & Torreja & & 27,99 & 360 & 333 & 0,00389 \\
\hline
\end{tabular}

Fuente: Elaboración propia. 
En las figuras 4 y 5 se presentan las curvas de secado encontradas para los cubos y torrejas de papaya sometidas a la razón de radiación de microondas $6 \times 12$, ciclos del control on/off de 6 segundos de encendido y 12 segundos de apagado.

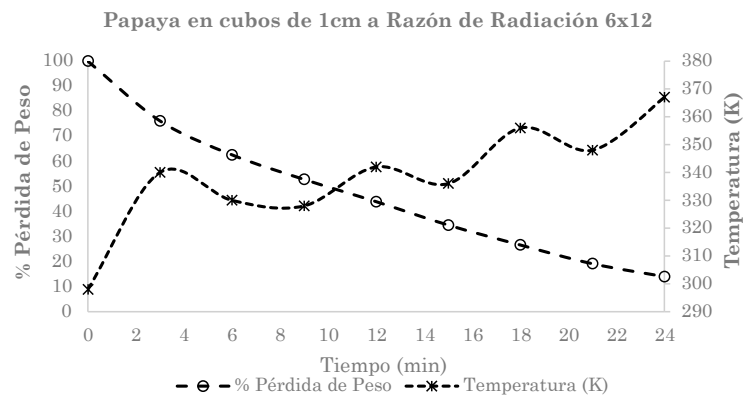

Fig. 4. Resultados de pérdida de peso y temperatura para cubos de $1 \mathrm{~cm}$ de papaya a razón de radiación de microondas de 6 × 12 . Fuente: Elaboración propia.

En la fig. 4 se observa que la temperatura se mantiene oscilante durante el proceso, lo cual se explica por la continua actividad de los fenómenos de transferencia de calor conductivos y por radiación, predominantemente. De igual forma, se observa una pérdida de peso instantánea considerable desde el inicio del suministro de radiación de microondas y una tendencia casi lineal de pérdida de peso una vez se alcanza una temperatura alrededor de los $60{ }^{\circ} \mathrm{C}$ que se mantiene durante todo el tiempo de operación sin deteriorar la muestra.

Papaya en torreja de 1cm a Razón de Radiación 6x12

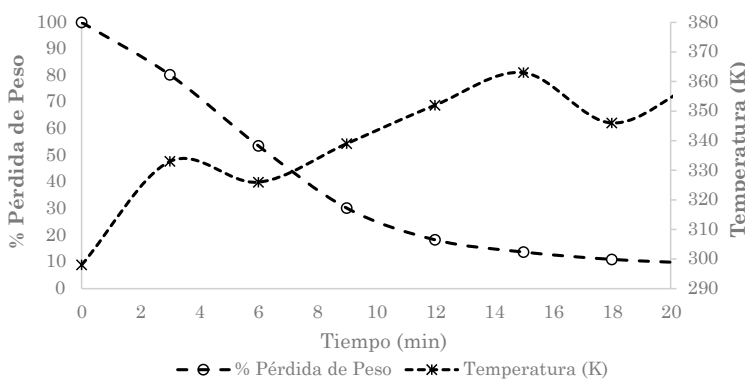

Fig. 5. Resultados de pérdida de peso y temperatura para torreja de $1 \mathrm{~cm}$ de alto de papaya a razón de radiación de microondas de 6 × 12 . Fuente: Elaboración propia.

En la fig. 5 se muestra, al compararlo con la fig. 4, la importancia del tamaño-geometría de la muestra en los procesos asistidos por razones de radiación de microondas. En ésta se visualiza un incremento de la temperatura más sostenido, lo que acelera inicialmente la transferencia de masa representada en la pérdida de peso, explicado por la retención de la energía térmica en el interior de la torreja que, posteriormente, luego de los 8 minutos de proceso, se atenúa en una tasa de pérdida de calor mínima.

\section{Conclusiones}

El control para el equipo diseñado consta de diferentes componentes electrónicos que se acoplan para manipular el encendido y apagado del magnetrón y la temperatura del material en tiempo real. Para su ejecución se realizaron pruebas con el montaje físico. Inicialmente se cambiaron los cristales, el hardware de la programación y otros componentes que no permitían el paso de voltaje a otros elementos por la corriente consumida, ya que el tiempo programado se retrasaba en comparación con la unidad de tiempo real. Para la medición de la temperatura en la superficie del proceso se empleó un sensor infrarrojo MLX90614ESF, el cual es capaz de medir la temperatura en un rango de entre $-70^{\circ} \mathrm{C}$ y $350^{\circ} \mathrm{C}$ y opera con un voltaje de alimentación de 5 V. Éste se instaló a una distancia de 0,30 $m$ con respecto a la posición del material dentro de la cavidad del horno microondas utilizado. Para llevar a cabo lo anterior, se modificó la tarjeta electrónica y la programación del microprocesador.

Con el objeto de que el magnetrón no oscilara con tanta frecuencia en cuanto a su encendido y apagado (afectando su vida útil $\mathrm{y}$, por ende, la del equipo), el control en modo automático se diseñó basado en un rango de temperatura que garantizara el funcionamiento de cada uno de los dos modos de funcionamiento desarrollados en este trabajo. Se realizaron pruebas técnicas para validar la operatibilidad del control diseñado y se pretende en futuros trabajos evaluar los rendimientos tanto operativos como energéticos de los procesos asistidos con microondas con este nuevo sistema de control propuesto.

El control diseñado está siendo probado en procesos de secado de frutas y extracción de aceites de semillas, de igual forma se pretende emplear para el proceso de producción de líquidos combustibles a partir de biomasa, fundición de vidrio, aceleración de reacciones químicas, entre otros. En los resultados alcanzados hasta el momento, se ha puesto en evidencia que las propiedades de cada muestra arrojan una temperatura y razón de radicación característica máxima en el procesamiento que minimiza los subproductos y efectos no deseados. Los factores que se han evaluado hasta el momento corresponden a la cantidad de material suministrado, su geometría (altura y ancho), ubicación espacial dentro de la cavidad, entre otros.

Con las pruebas realizadas se logró un máximo rendimiento que se evidencia en el proceso de secado de papaya con un $0,014 \mathrm{~kg} / \mathrm{kJ}$ y con una reducción del $86 \%$ de peso de la muestra procesada en la razón de radiación de 6 x 12 para una rodaja de 1 cm de espesor.

Los resultados muestran que el control on-off y de temperatura sí son un parámetro importante a estudiar en los procesos asistidos con microondas. 
De igual forma, se concluye que tanto la geometría como la cantidad de muestra en la cámara de procesamiento (secado) son factores que se deben evaluar o bloquear en los estudios de los procesos asistidos con microondas.

\section{FINANCIACIÓN}

Artículo de investigación científica derivado del proyecto de investigación titulado: "Estudio del secado de frutas asistido con microondas". Proyecto financiado por la Institución Universitaria ITSA (Soledad, Colombia), la Universidad Nacional de Colombia (Medellín, Colombia) y el Servicio Nacional de Aprendizaje SENA (Medellín, Colombia). Fecha de inicio: junio de 2015. Fecha de finalización: diciembre de 2017.

\section{Agradecimientos}

Los autores agradecen al aprendiz Brayan Stiven Arévalo del programa de Tecnología de Automatización Industrial del SENA quien apoyó el diseño de la tarjeta electrónica en Altium.

\section{- ReFERENCIAS}

[1] J. Bélanger, J. Jocelyn Paré, O. Poon, C. Fairbridge, S. $\mathrm{Ng}$, S. Mutyala y R. Hawkins, "Remarks on Various Applications of Microwave Energy," Journal of the Microwave Power and Electromagnetic Energy, 42 (4), 24-44, 2007. https://doi.org/10.1080/08327823.2007.11688597

[2] A. Altan y M. Maskan, "Microwave Assisted Drying for Short-Cut (Ditalini) Macaroni: Drying Characteristics and Effect of Drying Processes on Starch Properties," Food Research International, 38 (7), 787-796, 2005. https://doi.org/10.1016/j.foodres.2005.02.006

[3] Z. Li, V. Raghavan y V. Orsat, "Optimal power control strategies in microwave drying," Journal of Food Engineering, 99 (3), 263-268, 2010. https://doi.org/10.1016/j. jfoodeng.2010.02.024

[4] J. Zhang, M. Zhang, L. Shan, Z. Fang, "Microwave-Vacuum Heating Parameters for Processing Savory Crisp Bighead Carp (Hypophthalmichthys nobilis) Slices," Journal of Food Engineering, 79 (3), 885-891, 2007. https://doi.org/10.1016/j.jfoodeng.2006.03.008
[5] N. A. Rowson y N. Rice, "Desulphurisation of coal using low power microwave energy," Minerals Engineering, 3 (3-4), 363-368, 1990. https://doi.org/10.1016/0892 6875(90)90131-T

[6] J.-i. Hayashi, K. Oku, K. Kusakabe y S. Morooka, "The role of microwave irradiation in coal desulphurization with molten caustics," Fuel, 69 (6), 739-742, 1990. https://doi.org/10.1016/0016-2361(90)90039-S

[7] H. Zhang, X. Zheng y D. Su, "Postharvest Control of Blue Mold Rot of Pear by Microwave Treatment and Cryptococcus laurentii," Journal of Food Engineering, 77 (3), 539-544, 2006. https://doi.org/10.1016/j.jfoodeng.2005.06.066

[8] C. James, K. Barlow, S. James y M. Swain, "The Influence of Processing and Product Factors on the Quality of Microwave Pre-Cooked Bacon”. Journal of Food Engineering, 77 (4), 835-843, 2006. https://doi. org/10.1016/j.jfoodeng.2005.08.010

[9] M. García-Paternina, A. Alvis-Bermudez y C. GarcíaMogollón, "Evaluación de los Pretratamientos de Deshidratación Osmótica y Microondas en la Obtención de Hojuelas de Mango (Tommy Atkins)," Información tecnológica, 26 (5), 63-70, 2015.

[10] E. Holtz, L. Arhné, M. Rittenauer y A. Rasmuson, "Influence of dielectric and sorption properties on drying behaviour and energy efficiency during microwave convective drying of selected food and non-food inorganic materials," Journal of Food Engineering, 97 (2), 144-153, 2010. https://doi.org/10.1016/j.jfoodeng.2009.10.003

[11] E. Maji y J. Paucar, "Diseño e Implementación de un Sistema de Control Automático Centralizado en el Área de Molienda de Puzolana Miag de la Planta Cemento Chimborazo," Tesis de maestría, Escuela Superior Politécnica de Chimborazo, Riobamba, Ecuador, 2015.

[12] M. Alvarado-Bawab, "Estudio de secado en horno con tinuo microonda del carbón de Bijao-Córdoba," Tesis de maestría, Universidad Nacional de Colombia, Medellín, Colombia, 2012.

[13] P. González, "Control de la temperatura en sistemas de calentamiento por microondas," Tesis de doctorado, Universitat Politècnica de València, Valencia, España, 2016.

[14] M. Alvarado, J. Mejía, M. Vanegas Chamorro y L. Hernández, "Influential variables in coal batch microwave drying," 2012 IEEE International Symposium on Alternative Energies and Energy Quality (SIFAE), Barranquilla, 2012, pp. 1-6. https://doi.org/10.1109/ SIFAE.2012.6478899

[15] LG. Microwave Oven Service Manual. Model MS0746T [Internet]. Disponible en: <http://biz.lgservice. com>, [Acceso 15 de junio de 2015], 1-37, 2006. 